\title{
Working Memory-Related Neural Activity Predicts Future Smoking Relapse
}

\author{
James Loughead*,', E Paul Wileyto², Kosha Ruparel², Mary Falcone', Ryan Hopson², Ruben Gur ${ }^{2}$ and \\ Caryn Lerman*, I \\ 'Center for Interdisciplinary Research on Nicotine Addiction, Department of Psychiatry, University of Pennsylvania, Philadelphia, PA, USA; \\ ${ }^{2}$ Brain Behavior Laboratory, Department of Psychiatry, University of Pennsylvania, Philadelphia, PA, USA
}

\begin{abstract}
Brief abstinence from smoking impairs cognition, particularly executive function, and this has a role in relapse to smoking. This study examined whether working memory-related brain activity predicts subsequent smoking relapse above and beyond standard clinical and behavioral measures. Eighty treatment-seeking smokers completed two functional magnetic resonance imaging sessions (smoking satiety vs $24 \mathrm{~h}$ abstinence challenge) during performance of a visual N-back task. Brief counseling and a short-term quit attempt followed. Relapse during the first 7 days was biochemically confirmed by the presence of the nicotine metabolite cotinine. Mean percent blood oxygen level-dependent (BOLD) signal change was extracted from a priori regions of interest: bilateral dorsolateral prefrontal cortex (DLPFC), medial frontal/cingulate gyrus, posterior cingulate cortex (PCC), and ventromedial prefrontal cortex. Signal from these brain regions and additional clinical measures were used to model outcome status, which was then validated with resampling techniques. Relapse to smoking was predicted by increased withdrawal symptoms, decreased left DLPFC and increased PCC BOLD percent signal change (abstinence vs smoking satiety). Receiver operating characteristic analysis demonstrated $81 \%$ area under the curve using these predictors, a significant improvement over the model with clinical variables only. The combination of abstinence-induced decreases in left DLPFC activation and reduced suppression of PCC may be a prognostic marker for poor outcome, specifically early smoking relapse. Neuropsychopharmacology (20 I5) 40, I3II-1320; doi:I0.1038/npp.20 I4.3I8; published online 7 January 2015
\end{abstract}

\section{INTRODUCTION}

Maladaptive behaviors such as tobacco use, unhealthy diet, and physical inactivity have far reaching health and economic implications. Even with the best available interventions, many unhealthy behaviors remain difficult to modify. Emerging research suggests that impaired cognition, particularly executive cognitive function, plays a central role in failed attempts at behavior change (Filevich et al, 2012). Executive cognitive function refers to a core set of cognitive processes that support cognitive (self) control (Botvinick et al, 2001). Working memory, a central domain of cognitive control, is required to focus on goalrelated information and to sustain goal-directed behavior (Baddeley, 2003). Indeed, objective measures of working memory performance predict a variety of lifestyle behaviors, including smoking and maintenance of a healthy body weight (Gonzales et al, 2010; Hege et al, 2013; Patterson et al, 2010).

*Correspondence: Dr C Lerman or J Loughead, Department of Psychiatry, University of Pennsylvania, 3535 Market Street, Suite 4100, Philadelphia, PA 19104, USA, Tel: +I 2157467141 or + I 2157467292 , Fax: + I $2157467 \mid 40$,

E-mail: clerman@upenn.edu or loughead@upenn.edu

Received 30 July 2014; revised 21 October 2014; accepted 22 November 2014; accepted article preview online 3 December 2014
Functional magnetic resonance imaging (fMRI) studies have explored the effects of brief abstinence from smoking (eg, 24-72 h) on working memory and associated neural activation, using within-subject contrasts of smokers in the abstinent $v s$ smoking satiety states (Falcone et al, 2014; Jacobsen et al, 2007; Loughead et al, 2009). In addition to reducing working memory performance (Mendrek et al, 2006; Myers et al, 2008), brief abstinence is associated with reduced working memory-related activation in the dorsolateral prefrontal cortex (DLPFC) and medial frontal/ cingulate gyrus (MF/CG; Falcone et al, 2014; Loughead et al, 2010), decreased working memory-related suppression of activity in regions within the default mode network (posterior cingulate cortex (PCC), ventromedial prefrontal cortex (vmPFC); Beaver et al, 2011; Falcone et al, 2014; Loughead et al, 2010), and dysregulated inter-network functional connectivity during task performance and at rest (Jacobsen et al, 2007; Lerman et al, 2014; Sutherland et al, 2013). FDA-approved medications, such as varenicline and bupropion, can reverse abstinence-induced working memory deficits and related neural activation in studies of abstinent smokers tested during medication vs placebo (Loughead et al, 2010; Perkins et al, 2013). To our knowledge, no study has examined whether working memoryrelated brain signal predicts relapse to smoking.

We tested the hypothesis that abstinence-induced changes in working memory-related neural activation 
would predict the likelihood of early smoking relapse (biochemically confirmed in the first 7 days of a quit attempt) above and beyond standard clinical and behavioral measures. Prior work suggests that regions within the brain's executive control network (eg, DLPFC, MF/CG) are vital for cognitive control (Fassbender et al, 2004; Krain et al, 2006), whereas regions within the default mode network (eg, PCC, vmPFC) are involved in self-referential and stimulus-independent thought processes that can compete with executive control functions (Gusnard et al, 2001; Smith et al, 2009). Given the importance of outwardly focused attention and cognitive control in smoking behavior change (Ashare et al, 2013b; Kollins et al, 2013; Sutherland et al, 2012), we expected that short-term relapse would be predicted by reduced activation in DLPFC and MF/CG, and less suppression of activation in PCC and vmPFC, as assessed during a pre-quit abstinence challenge (vs smoking satiety).

\section{MATERIALS AND METHODS}

\section{Participants}

Treatment-seeking smokers aged 18-65 years who reported smoking $\geqslant 10$ cigarettes/day for $\geqslant 6$ months were recruited through mass media $(N=80)$. Participants provided written informed consent and completed a physical examination including a breath alcohol test, urine drug screen, and urine pregnancy test (females). Persons with a history of DSM-IV Axis I psychiatric or substance disorders (except nicotine dependence) assessed with the Mini International Neuropsychiatric Interview (Sheehan et al, 1998) and those taking psychotropic medications were excluded. Exclusion criteria also included: current use of chewing tobacco, snuff, or smoking-cessation products; pregnancy, planned pregnancy or breastfeeding; history of brain injury; left handedness; presence of fMRI contraindicated material in the body; low or borderline intelligence $(<90$ score on Shipley Institute of Living Scale; Zachary, 1986); and any impairment that would prevent task performance. Eligible participants completed the six-item Fagerström Test for Nicotine Dependence (Heatherton et al, 1991). The University of Pennsylvania Institutional Review Board approved all procedures.

\section{Functional Brain Imaging Sessions}

Two blood oxygen level-dependent (BOLD) fMRI sessions were acquired in counterbalanced order: (1) smoking satiety and (2) $24 \mathrm{~h}$ abstinence challenge. The $24-\mathrm{h}$ period was selected because the majority of relapses occur in the first day of a quit attempt (Piasecki, 2006). Imaging sessions were separated by $\sim 2$ weeks. Subjects were to refrain from alcohol and other drugs $24 \mathrm{~h}$ before the imaging session and those with a positive drug screen, a breath alcohol test $>0.01$, or a breath carbon monoxide (CO) test $>9 \mathrm{ppm}$ (abstinence challenge condition only) were excluded. Before imaging, participants completed the Minnesota Nicotine Withdrawal Scale (MNWS; Hughes and Hatsukami, 1986), Questionnaire of Smoking Urges (QSU-Brief; Cox et al, 2001), and the Positive and Negative Affect Schedule (PANAS; Watson et al, 1988). For the smoking satiety condition, participants smoked immediately before scanning to standardize exposure ( $\sim 30 \mathrm{~min}$ prior to task performance).

Working memory was assessed during BOLD imaging with a visual N-back task (Ragland et al, 2002) used in our prior research (Loughead et al, 2010; Loughead et al, 2009). This task presents complex geometric figures (fractals) under four conditions: 0-back, 1-back, 2-back, and 3-back. In the 0-back condition, participants respond with a button press to a specified target fractal; for the 1-back condition, participants respond if the current fractal was identical to the previous one; for the 2-back condition, if the current fractal was identical to the item presented two trials back; etc. No response was required for nontargets. Images were presented for $500 \mathrm{~ms}$ with an interstimulus interval of $2500 \mathrm{~ms}$. The task began with a $48 \mathrm{~s}$ baseline rest period (fixation point) of which the first $24 \mathrm{~s}$ was discarded to ensure the MRI signal reached steady state. Each condition was presented three times in 20-trial blocks (33\% targets; $60 \mathrm{~s})$. Blocks were presented in order of increasing memory load for one set, after which conditions were presented pseudo-randomly; visual instructions $(9 \mathrm{~s})$ preceded each block to indicate the upcoming condition. Equivalent forms with unique stimuli were used and task version order was counterbalanced.

\section{fMRI Data Acquisition}

BOLD fMRI was acquired with a Siemens Trio 3T (Erlangen, Germany) system using a whole-brain, single-shot gradientecho echoplanar sequence with the following parameters: $\mathrm{TR} / \mathrm{TE}=3000 / 30 \mathrm{~ms}, \mathrm{FOV}=220 \mathrm{~mm}$, matrix $=64 \times 64$, slice thickness $/$ gap $=3.4 / 0 \mathrm{~mm}, 48$ slices, effective voxel resolution of $3.4 \times 3.4 \times 3.4 \mathrm{~mm}$. RF transmission utilized a quadrature body coil, and reception used a 32-channel head coil. Prior to BOLD fMRI, 5-min magnetizationprepared, rapid acquisition gradient echo T1-weighted image (MPRAGE, TR $1620 \mathrm{~ms}$, TE $3.87 \mathrm{~ms}$, FOV $50 \mathrm{~mm}$, matrix $192 \times 256$, effective voxel resolution of $1 \times 1 \times 1 \mathrm{~mm}$ ) was acquired for anatomic overlays of functional data and to aid spatial normalization to standard atlas space.

\section{Smoking Cessation Procedures}

Approximately 1-2 weeks after imaging, participants took part in a standardized counseling session during which they discussed strategies for quitting (including nicotine fading, identifying and managing triggers, problem solving, relapse prevention, and stress management) with a trained smoking cessation counselor (Lerman et al, 2004). During this counseling session, participants set a target quit date (TQD) to occur $\sim 1$ week later and received a self-help booklet to take home (UDHHS, 2006). Participants completed a brief in-person visit on the TQD, which included a 20-min booster counseling session to reinforce and refine strategies discussed at the initial session. See 'Outcome Measure' below for the assessment of subsequent quit status.

\section{Image Preprocessing}

BOLD time series data were preprocessed and analyzed by standard procedures using fMRI Expert Analysis Tool 
(FEAT version 5.98) of FSL (FMRIB's Software Library, Oxford, UK). Single-subject preprocessing included skull stripping using the FSL Brain Extraction Tool (Smith, 2002), slice time correction, motion correction to the median image using MCFLIRT (Jenkinson and Smith, 2001), high pass temporal filtering (138s), spatial smoothing using a Gaussian kernel (6 mm full-width at half-maximum, isotropic), and mean-based intensity normalization of all volumes with the same multiplicative factor. The median functional volume was coregistered to the anatomical T1-weighted structural volume and transformed into a standard anatomical space (T1 MNI template), using FLIRT (Jenkinson et al, 2002; Jenkinson and Smith, 2001). Transformation parameters were later applied to statistical maps for group-level analyses.

\section{ROI Definition}

As noted above, prior work with the visual N-back task by our group (Ashare et al, 2013b; Falcone et al, 2014; Lerman et al, 2014; Loughead et al, 2009, 2010) and others (Owen et al, 2005; Satterthwaite et al, 2013) has identified three task-active (right DLPFC, left DLPFC, MF/CG) and two task-negative (PCC, vmPFC) regions sensitive to abstinence challenge. ROI masks were functionally defined using the main effect of memory load (controlling for condition) in a whole-brain repeated measures condition (smoking satiety, abstinence challenge) by memory load (N-back level) ANOVA. Seven-day quit outcome was not included in this analysis. The resulting activation map was thresholded for family-wise error correction at $p \leqslant 0.001 \quad(Z \geqslant 5.87)$ to account for five a priori ROIs and then segmented by clusters $\geqslant 150$ voxels using a watershed algorithm implemented in MATLAB (The Mathworks, Natick, MA). The five functionally defined a priori ROI masks (right DLPFC, left DLPFC, MF/CG, vmPFC, and PCC) were transformed to native subject space using parameters obtained in the time series analysis (Table 1). Each participant's mean percent signal change was calculated for the memory load conditions in each ROI and exported for further statistical analysis.

Table I Functional Regions of Interest Identified by Main Effect of Memory Load in a Whole-brain Repeated Measures ANOVA

\begin{tabular}{llrrrrrr}
\hline Region $^{\mathbf{a}}$ & Hem $^{\mathbf{b}}$ & Count $^{\mathbf{c}}$ & $\mathbf{m m}^{\mathbf{3}}$ & $\mathbf{Z}-\mathbf{m a x}^{\mathbf{d}}$ & $\mathbf{X}^{\mathbf{e}}$ & $\mathbf{Y}$ & $\mathbf{Z}$ \\
\hline MF/CG & R/L & 422 & 16586 & 14.87 & 0 & 16 & 48 \\
DLPFC & $\mathrm{L}$ & 197 & 7743 & 12.13 & -44 & 24 & 26 \\
VmPFC & R/L & 196 & 7703 & 12.05 & 0 & 42 & -6 \\
DLPFC & R & 134 & 5266 & 8.55 & 42 & 32 & 28 \\
PCC & R/L & 41 & 1608 & 8.05 & 0 & -50 & 26
\end{tabular}

Abbreviations: DLPFC, dorsolateral prefrontal cortex; MF/CG, medial frontal/ cingulate gyrus; PCC, posterior cingulate cortex; vmPFC, ventromedial prefrontal cortex.

a'Significant clusters > 150 contiguous voxels.

${ }^{\mathrm{b}}$ Hem $=$ cerebral hemisphere.

'Voxel count (3.4 mm isometric).

dZ-max peak activation for cluster.

${ }^{\mathrm{e}}$ Coordinates (mm) from Talairach and Tournoux (1988).

\section{Outcome Measure}

The smoking cessation outcome measure was short-term abstinence; specifically, the ability to remain completely abstinent from smoking (not even a puff) for 7 days following the TQD. Participants completed a monitoring visit on day 7 , during which smoking behavior was assessed using a timeline follow-back method (Brown et al, 1998) and biochemically confirmed using NicAlert urine test strips (Nymox Pharmaceutical Corporation, Hasbrouck Heights, NJ). NicAlert test strips utilize an immunochromatographic assay to provide a semiquantitative measure of the concentration of cotinine (the primary metabolite of nicotine) in urine. Results appear as categorical levels of usage (range: 0-6). Following manufacturer guidelines, NicAlert results of level 2 or below (corresponding to a urine cotinine concentration of $\leqslant 100 \mathrm{ng} / \mathrm{ml}$ ) were required to biochemically confirm abstinence; levels 3-6 were considered indicative of current smoking. The 7-day monitoring period was chosen because $50-75 \%$ of smokers who relapse do so within the first 7 days of a quit attempt (Hughes et al, 2004). Further, this measure is a well-validated indicator of long-term smoking status; continuous abstinence for the first week of a quit attempt is a highly significant predictor of abstinence at 6 months following the TQD (Ashare et al, 2013a). Sixty-one smokers relapsed and 19 quit successfully for this period. Of this sample, data from four relapsed and four quit participants were excluded from analysis owing to poor imaging data quality (root mean square mean relative motion $>0.4$, total non-responses $>66 \%$ ).

\section{Statistical Analysis}

Descriptive statistics were obtained for all variables. To examine expected abstinence challenge effects, mean percent signal change was modeled using regression with subject-level random effects, and estimated using maximum likelihood techniques (Stata xt-reg; Stata Corporation, College Station, TX) with mixed effects (Gaussian model). The models of abstinence challenge effects on BOLD signal included terms for the main effects of condition, categorical memory load and relevant covariates (age, sex, FTND score, Shipley IQ score, and condition order). Models of abstinence effects on smoking measures and task performance used similar regression models (subject-level random effects, maximum likelihood techniques with mixed effects), which utilized the measure of interest (CO reading, MNWS, QSU-B, N-back accuracy, or N-back correct response time) as the independent variable and included terms for main effects of condition, categorical memory load (for task performance) and relevant covariates (age, sex, FTND score, Shipley IQ score, and condition order). Multi-level mixed effects logistic regression (Stata xtmelogit) was used to examine whether BOLD signal in the $a$ priori regions predicted performance outcomes (N-back accuracy, N-back correct response time), controlling for condition and categorical memory load.

Predictive models. Forward stepwise regression with a probability of 0.2 for removal and 0.1 for entry was used to select predictors for a logistic regression model (STATA logistic) of dichotomized 7-day relapse. Sex, age, and 
nicotine dependence level (Heatherton et al, 1991) were entered as baseline candidate predictors of relapse (Gourlay et al, 1994; Hymowitz et al, 1997; Japuntich et al, 2011; Sweitzer et al, 2013). Change scores (abstinence challenge minus smoking satiety) for paired data collected during abstinence challenge (MNWS, QSU-Brief, PANAS Positive, PANAS Negative, task performance (accuracy and response time), right DLPFC, left DLPFC, MF/CG, vmPFC, and PCC) were also included in the model. Prior work has demonstrated that nicotine abstinence effects are induced at the highest working memory load (3-back level; Loughead et al, 2009, 2010). Therefore, we examined task performance and BOLD signal acquired during performance of 3-back trials (minus BOLD signal acquired during performance of 0 -back trials to account for task-related cognitive processes other than working memory). We required age and nicotine dependence to be entered based on clinical relevance (Hymowitz et al, 1997; Sweitzer et al, 2013); sex was nonsignificant, and allowed to dropout.

Following logistic regression, receiver operating characteristic (ROC) analysis was used to assess the predictive potential of the models (Steyerberg, 2008). Classification models, based on linear scores, selected a cut point for separating predicted classes. ROC curve analysis varied the cut point across the range of data, plotting true-positive rate against the false-positive rate for each increment. Estimates of predictive accuracy are expressed as area under curve (AUC). The ROC curve is a plot of the sensitivity vs 1-specificity of a classification system, represents the accuracy of the system, and provides a comparable metric across experiments (Bradley, 1997). Following literature standards (Fawcett, 2003; Harrell et al, 1996), we consider ROC curve AUC of $0.50-0.60$ as indicating prediction at chance; $0.60-0.70$ as indicating poor prediction; $0.70-0.80$ fair prediction; $0.80-0.90$ good prediction; and $0.90-1.0$ excellent prediction.

Two resampling methods were used to validate predictive models. First, bootstrap procedures were used to create 1000 replicates of the data with records chosen randomly with replacement. Stepwise model regression (see above) was applied to each replicate to assess model uncertainty (Austin and Tu, 2004b). The frequency of a variable's inclusion in a model can be summarized and variables selected most often are considered to be the most robust (ie, least influenced by outliers and noise). To examine the model's potential for prediction in new cases, we performed leave-one-out cross-validation (LOOCV) using standard jackknife procedures (Steyerberg, 2008).

\section{Exploratory Analysis}

In a second validation analysis, a sub-sample of relapsers was selected and matched for age and sex with the group of quitters, resulting in a sample of 15 pairs. Matching was conducted without replacement, and there were no cases where more than one relapser was matched to a quitter. An exploratory whole-brain condition (abstinence challenge $v s$ smoking satiety) by smoking cessation group (relapse $v s$ quit) voxelwise $2 \times 2$ ANOVA at the 3-back level (minus 0 -back) was conducted to identify novel regions sensitive to quit-group status. Resulting statistical maps were corrected for multiple comparison using random field theory with group maps cluster corrected at $Z>1.96$ and probability of spatial extent $p<0.05$.

\section{RESULTS}

\section{Baseline Sample Characteristics and Abstinence Challenge Effects}

Of 72 participants included in the analysis, $44.4 \%$ were female and $38.9 \%$ were Caucasian. The mean age was 41.9 years (SD 14.4), mean FTND score was 4.8 (SD 1.8), and mean Shipley IQ score was 103.5 (SD 8.4). Mean CO, MNWS, QSU-B, and N-back response times all showed significant condition effects, indicating compliance with the abstinence requirements (reduced $\mathrm{CO}$ readings during abstinence challenge), more severe withdrawal symptoms (MNWS) and craving (QSU-B), and slower response times on the N-back task during abstinence challenge compared with smoking satiety ( $\mathrm{ps} \leqslant 0.05$, Table 2 ). There was no significant condition effect on N-back accuracy. Abstinence challenge effects were observed on BOLD signal for MF/CG $(\beta=-0.17,95 \%$ confidence interval $(\mathrm{CI})-0.26$ to -0.08 ,

Table 2 Smoking Characteristics and Performance Across Condition (Abstinence Challenge, Smoking Satiety) and Group (Quit vs Relapse) $(N=72)$

\begin{tabular}{|c|c|c|c|c|}
\hline \multirow[t]{2}{*}{ Measure } & \multicolumn{2}{|c|}{ Quit } & \multicolumn{2}{|c|}{ Relapse } \\
\hline & Smoking satiety & Abstinence challenge & Smoking satiety & Abstinence challenge \\
\hline Carbon monoxide $(\mathrm{ppm})^{\mathrm{a}}$ & $26.4(17.6)$ & $3.7(2.6)$ & $27.8(12.5)$ & $3.8(2.1)$ \\
\hline QSU-brief ${ }^{\mathrm{a}}$ & $23.4(8.7)$ & $39.9(13.0)$ & $23.1(11.9)$ & $44.9(16.1)$ \\
\hline MNWS $^{a}$ & $5.1(5.1)$ & $9.3(5.4)$ & $3.6(4.3)$ & | I.8 (8.6) \\
\hline PANAS-negative affect & $12.1(3.1)$ & | $4.7(4.7)$ & $12.2(3.7)$ & $16.0(7.0)$ \\
\hline PANAS-positive affect & $32.1(7.8)$ & $31.6(6.6)$ & $32.2(8.2)$ & $30.0(10.0)$ \\
\hline N-back total correct & $50.1(6.8)$ & $49.0(7.6)$ & $46.2(8.4)$ & $45.0(7.7)$ \\
\hline N-back reaction time ${ }^{a}$ & $655.6(|| 6.5)$ & $656.8(109.2)$ & $662.1(112.6)$ & $674.0(111.8)$ \\
\hline
\end{tabular}

Abbreviations: MNWS, Minnesota Nicotine Withdrawal Scale; ppm, parts per million; QSU-Brief, Questionnaire of Smoking Urges.

Values are mean (SD).

a Significant effect of abstinence challenge in overall sample $(p<0.05)$. 
$p<0.001)$, right DLPFC $(\beta=-0.13,95 \%$ CI -0.26 to $-0.01, p=0.039)$, left DLPFC $(\beta=-0.17,95 \%$ CI -0.31 to $-0.03, p=0.016)$, and PCC $(\beta=-0.21,95 \%$ CI 0.05 to $0.37, p=0.008$ ), consistent with the effects we previously reported in an analysis of the abstinence challenge data for a subset of these participants (Falcone et al, 2014) and in prior studies (Loughead et al, 2009, 2010). Specifically, in the task-positive regions (MF/CG, left DLPFC, and right DLPFC), abstinence challenge ( $v s$ smoking satiety) was associated with reduced activation during task performance; whereas in the task-negative PCC, abstinence challenge was associated with less deactivation during task performance compared with smoking satiety. There was no significant effect of abstinence challenge on BOLD signal in the vmPFC. We examined associations between BOLD signal, accuracy, and correct response times, controlling for condition and memory load. BOLD signal in right and left DLPFC was positively associated with accuracy (left DLPFC: $\mathrm{OR}=1.13,95 \% \mathrm{CI}=1.04$ to $1.23, p=0.006$; right DLPFC: $\mathrm{OR}=1.09,95 \% \mathrm{CI}=1.01$ to $1.18, p=0.04)$. There was no association between correct response time and BOLD signal in any region ( $\mathrm{ps}>0.05)$.

\section{Predictive Models for Quitting vs Relapse}

Forward stepwise regression retained MNWS, left DLPFC, and PCC as predictors (Figure 1) with participant age also contributing to the model. Relapse (a reduced odds of quitting success) was predicted by older age $(\mathrm{OR}=1.07$, $95 \%$ CI 1.01-1.13, $p=0.02)$ and greater withdrawal change score (MNWS abstinence challenge minus smoking satiety, $\mathrm{OR}=1.22,95 \%$ CI $1.01-1.37, p=0.04)$. BOLD percent signal

\section{Abstinent minus Smoking Difference}

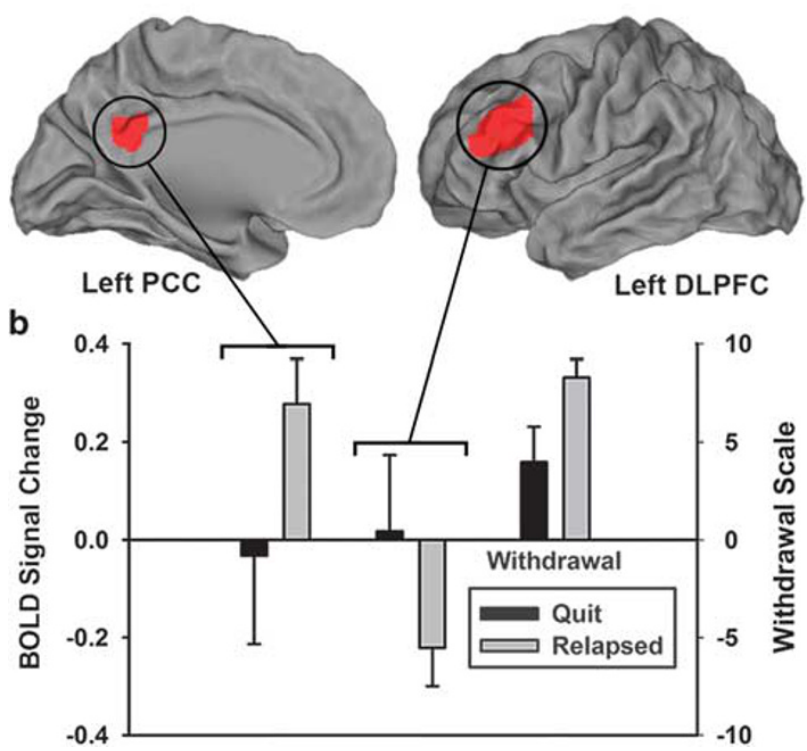

Figure I (a) Visualization of functionally defined $\mathrm{N}$-back ROls in the left dorsolateral prefrontal cortex (DLPFC) and posterior cingulate cortex (PCC). (b) Forward stepwise logistic regression retained the left DLPFC, left PCC, and withdrawal scores. Greater abstinence-induced change in withdrawal (increase), left DLPFC percent signal change (reduced activation), and PCC percent signal change (less deactivation) were predictive of relapse. change from two ROIs added significantly to the predictive model of relapse; reduced left DLPFC activation in abstinence challenge ( $v s$ smoking satiety) predicted greater odds of relapse (quitting failure) $(\mathrm{OR}=0.14$ per standardized increase in percent signal change, 95\% CI 0.03-0.74, $p=0.02$ ), and less deactivation in the PCC (task-negative region) during abstinence challenge ( $v s$ smoking satiety) predicted increased odds of relapse $(\mathrm{OR}=3.45$ per standardized increase in percent signal change, 95\% CI 1.05-11.32, $p=0.04)$.

The predictive value of the relapse model was examined using ROC curve analysis. The full model (age, FTND, MNWS, QSU-B, MF/CG, right DLPFC, left DLPFC, PCC) produced a relatively high AUC of $81 \%$ corresponding to $87.5 \%$ correct prediction rate at the optimal cut point (the point of optimal model performance; Figure 2). This model represents a significant improvement over clinical predictors (age, FTND) alone (AUC 67\%, $\mathrm{X}^{2}(1)=4.06, p<0.05$ ). We also examined an intermediate model, using clinical and withdrawal scores (age, FTND, MNWS, QSU-B), which achieved an AUC of 73\%, which did not differ significantly from the full or clinical models (AUC $67 \%, \mathrm{X}^{2}(1)=1.74$, $p<0.19$ ).

The full model was validated using two resampling methods. Bootstrapping (Austin and Tu, 2004a) generated 1000 replicates of the data and conducted model selection on each replicate. Accounting for age and nicotine dependence, the most frequent variables selected were withdrawal, left DLPFC, and PCC-the same variables identified by stepwise procedures. LOOCV further evaluated the potential inflation of model performance. Compared with the $81 \%$ AUC for the full model, LOOCV procedures yielded $71 \%$ AUC, corresponding to $82.7 \%$ correct prediction rate at the optimal cut point. Descriptively, performance of the model under LOOCV fell in the 'fair prediction' range in contrast to 'good prediction', demonstrated by the training data. Shrinkage of coefficients obtained during the crossvalidation of the ROC analyses is expected and falls within the typical range (Whelan and Garavan, 2014). The full

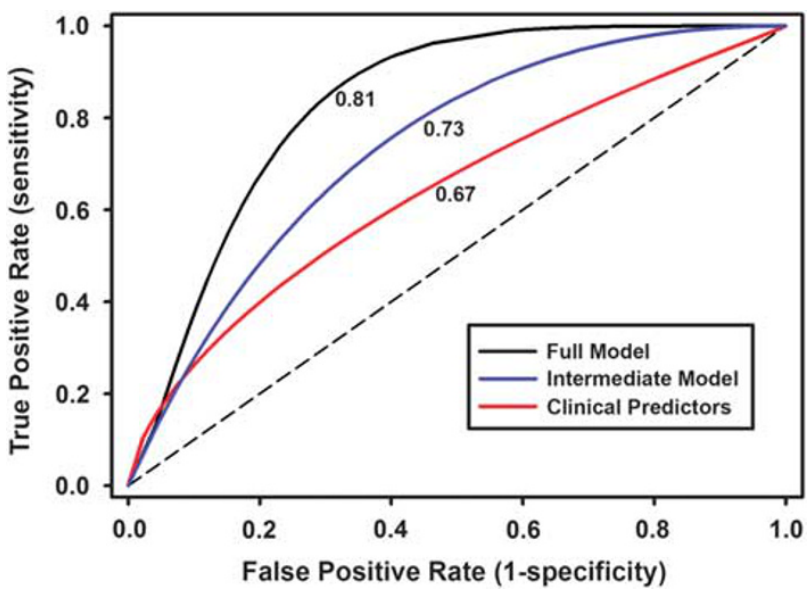

Figure 2 ROC curves for three predictive models of 7-day quit status. The full model (black) includes clinical, withdrawal and brain variables, yielding an AUC of $81 \%$. Clinical predictors alone (red) achieved an AUC of $67 \%$ and the intermediate model, using only clinical and withdrawal scores, an AUC of $73 \%$ (blue). 
Table 3 Condition (Smoking Satiety, Abstinence Challenge) by Group (Quit, Relapse) Whole-brain Interaction Results $(Z>1.96, p<0.05)$ for Matched Sample $(N=30)$

\begin{tabular}{|c|c|c|c|c|c|c|c|c|}
\hline Region (Brodmann) ${ }^{a}$ & $\mathrm{Hem}^{\mathrm{b}}$ & $p$-value & Count & $\mathrm{mm}^{3}$ & Z-max ${ }^{c}$ & $X(\mathrm{~mm})^{d}$ & $\mathbf{Y}(\mathbf{m m})$ & $Z(\mathbf{m m})$ \\
\hline DLPFC (BA 8) & $\mathrm{R}$ & $<0.0001$ & 87 & 3419 & 3.37 & 38 & 20 & 50 \\
\hline DLPFC (BA 8) & $L$ & $<0.0001$ & 70 & 2744 & 3.17 & -52 & 10 & 34 \\
\hline MF/CG (BA 8) & $\mathrm{R}$ & $<0.0005$ & 48 & 1886 & 2.85 & 4 & 24 & 50 \\
\hline
\end{tabular}

${ }^{a}$ Significant clusters $Z \geqslant 1.96$ and clusters probability $p<0.05$.

bHem $=$ cerebral hemisphere.

'ZZ-max values represent peak activation for cluster.

'Estimated Brodmann's areas and coordinates from Talairach and Tournoux (1988).

model LOOCV AUC (71\%) exceeds the rule-of-thumb cutoff for acceptable discrimination (an AUC of at least 70\%; Hosmer and Lemeshow, 2000). Notably, when subjected to LOOCV procedures both the clinical and intermediate model AUC fell in the 'chance' range.

The exploratory whole-brain condition (abstinence challenge, smoking satiety) by group (relapse, quit) voxelwise $2 \times 2$ ANOVA in the matched sample $(N=30)$ identified significant interaction effects in three clusters (Table 3 ). These clusters overlapped considerably with our functionally defined $a$ priori ROIs in the left DLPFC, right DLPFC, and MF/CG (Figure 3a). An interaction effect in the PCC was seen at an uncorrected threshold $(p<0.05)$. For the MF/CG and right DLPFC clusters, percent signal change did not differ between the quit and relapsed groups during the smoking satiety condition; however, when abstinent, MF/CG and right DLPFC signal decreased in relapsers and increased in quitters (Figure $3 \mathrm{~b}$ ). In the left DLPFC, the quit group ( $v s$ relapse) activated less during the smoking satiety condition and this pattern reversed under abstinence challenge (quit group showed increased signal). Consistent with the ROI-based analysis described above, abstinence challenge reduced signal in all clusters for the relapse group.

\section{DISCUSSION}

These data suggest that disruptions in working memoryrelated neural activation during early abstinence may distinguish successful quitters from those who fail. Specifically, decreased left DLPFC and increased PCC percent BOLD signal change and increased withdrawal symptoms (in the abstinence challenge $v s$ smoking satiety conditions) predicted subsequent relapse. Further, an exploratory whole-brain analysis revealed clusters overlapping with the functionally defined a priori ROIs in the DLPFC and MF/CG; however, the effect in the PCC was seen at an uncorrected threshold only $(p<0.05)$. Both analyses are consistent in that the relapse group is characterized by a reduction in left DLPFC during abstinence challenge $v s$ smoking satiety. In terms of model prediction, receiver operator curve analysis demonstrated $81 \%$ AUC for predicting smoking relapse using these predictors, a significant improvement over the model with clinical variables only. Cross-validation results revealed an expected degree of shrinkage; specifically, we observed a 10 percentage point change (from 0.81-0.71), which falls within an expected range for both model shrinkage (see Whelan and Garavan, 2014) and discrimination (an AUC of at least 0.70; Hosmer and Lemeshow, 2000).

The current findings supporting the predictive value of working memory-related DLPFC BOLD signal change during abstinence challenge are consistent with emerging data on cognitive neuroscience and behavior change. The DLPFC is at the core of the brain's cognitive control network, supporting sustained attention to and memory of long-term goals, and goal-directed decision-making (MacDonald et al, 2000; Niendam et al, 2012). Indeed, when the DLPFC is engaged, activation in reward-sensitive regions (eg, nucleus accumbens, ventral striatum) can be shifted away from the drive to obtain immediate gratification in the form of smoking or eating unhealthy foods (Hare et al, 2009; Kouneiher et al, 2009; Nestor et al, 2011). Likewise, studies of resting state functional connectivity have shown that activity in the executive control network (which includes DLPFC) is inversely correlated with activation in the default mode network (Fox et al, 2005). The PCC is a central hub in the default mode network (AndrewsHanna et al, 2010; Raichle et al, 2001), and suppression of activation (or deactivation) is integral to shifting attention away from stimuli irrelevant to a goal directed behavior (eg, quitting). Stimuli that are disruptive to an abstinent smoker include external environmental cues as well as internal processes such as craving and intrusive thoughts about wanting to smoke (Ashare et al, 2013b; Lerman et al, 2014).

Dopamine levels in the DLPFC are modulated, in part, by signaling at both $\alpha 4 \beta 2$ and $\alpha 7$ nicotinic acetylcholine receptors (nAChRs) in the ventral tegmental area (Arnsten and Jin, 2014; Nocente et al, 2013). Consistent with these molecular mechanisms and with the present data, we have shown that varenicline, a partial agonist at $\alpha 4 \beta 2$ and full agonist at $\alpha 7 \mathrm{nAChRs,}$ restores working memory performance and increases DLPFC activity during abstinence challenge compared with placebo during smoking abstinence (Loughead et al, 2010). In animal models, $\alpha 7 \mathrm{nAChRs}$ modulate both dopamine and NMDA receptor function in the DLPFC (Croxson et al, 2011) and working memory deficits caused by ketamine can be restored with an $\alpha 7$ agonist (Brozoski et al, 1979). These findings suggest the hypothesis that modulators of $\alpha 4 \beta 2$ and/or $\alpha 7 \mathrm{nAChRs}$ could be particularly beneficial for smoking cessation among smokers who exhibit larger abstinence-induced 
a

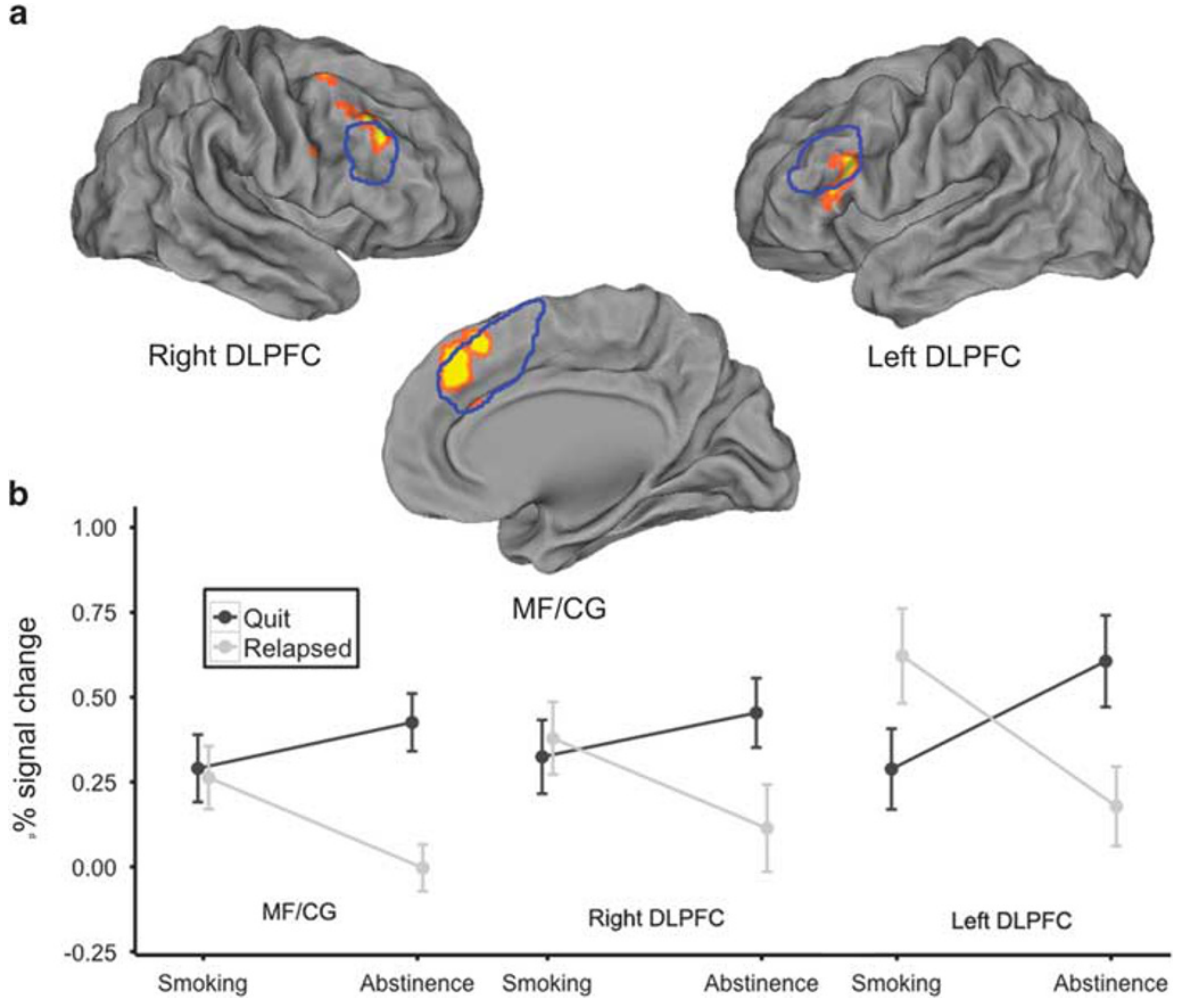

Figure 3 (a) Whole-brain condition (abstinence challenge, smoking satiety) by group (relapse, quit) voxelwise ANOVA interaction effect. Clusters (orange/yellow) are corrected for multiple comparison $(Z>1.96$ and probability of spatial extent $p<0.05)$ and superimposed outlines (blue) delineate a priori ROls. (b) MF/CG and right DLPFC clusters (see above) show comparable activation under the smoking satiety condition in both groups. There is greater activation in the left DLPFC in the smoking satiety condition for the relapse group (vs quit). Under abstinence, challenge signal in all clusters decreases for the relapse group and increases for the quit group.

working memory deficits. Dopamine levels in DLPFC are also influenced by levels of the enzyme catechol$O$-methyltransferase (COMT), and the COMT val158met variant has been shown to modulate working memory performance and associated BOLD signal change (Ashare et al, 2014; Loughead et al, 2009). Smokers with the val/val genotype (associated with a more active enzyme, resulting in decreased prefrontal dopamine levels) demonstrated impaired performance on the N-back task accompanied by reduced activation in the DLPFC during abstinence challenge compared to smoking (Loughead et al, 2009). However, pharmacologic inhibition of COMT with shortterm tolcapone treatment had minimal effects on smoking behavior and no effects on working memory-related BOLD signal in either the DLPFC or PCC (Ashare et al, 2013b). Future pharmacoimaging trials for smoking cessation incorporating working memory testing and fMRI may be useful to evaluate whether increased DLPFC activity, and possibly reduced PCC activity, serves as an intermediate biomarker of smoking cessation treatment efficacy (Bough et al, 2013).

Nonpharmacologic interventions that augment DLPFC function, support neuronal plasticity, and improve executive cognitive control may also have utility for smoking cessation. Cognitive exercise training, real-time fMRI feedback, and transcranial direct current brain stimulation (tDCS) have shown some promising initial results, supporting the enhancement of both working memory performance and DLPFC function (Dresler et al, 2013; Snowball et al, 2013; Zhang et al, 2013). However, evidence for beneficial effects on smoking behavior from preliminary studies is mixed (Sheffer et al, 2013; Wing et al, 2013). Other recent data suggest that concurrent treatment with both cognitive training and DLPFC-targeted tDCS may be most profitable (Segrave et al, 2014).

Although the current study reveals the significant contribution of neural activity to prospectively measured smoking relapse, the relatively small sample of successful quitters is a limitation. The low success rate is a consequence of using brief behavioral counseling without medication to support the quit attempt. However, a majority of quit attempts take place under similar conditions, making our results representative of the natural environment. Given the low success rates for unaided quitting, our study focused on a short-term (7 day) relapse measure rather than long-term cessation. It should be noted, however, that this measure is highly predictive of long-term quitting success (Ashare et al, 2013a). A replication sample is required to validate the model; however the observed shrinkage in AUC with the LOOCV procedure suggests that our results are not likely to represent inflated predictive power.

Although the broad implementation of neuroimaging assessment is not currently clinically or economically feasible, the mechanisms identified are potential targets for improved assessment instruments to assist in personalized intervention strategies. In addition to guiding 
assessment, predictive models can identify therapeutic targets for pharmacotherapies or neuroscience-based nonpharmacologic interventions to promote smoking cessation. Regardless of therapeutic approach, models sensitive to relapse risk can guide the refinement of existing interventions and provide early signal regarding the efficacy of novel tobacco dependence treatments.

\section{FUNDING AND DISCLOSURE}

Dr Lerman has served as a consultant and/or received research funding from Pfizer. Dr Gur has received compensation for professional services from US Courts. The remaining authors declare no conflict of interest.

\section{ACKNOWLEDGEMENTS}

This research was supported by grants from the National Cancer Institute and National Institutes on Drug Abuse (P50CA143187 and R03DA027438), and the Commonwealth of Pennsylvania Department of Health. The Department of Health disclaims responsibility for any analyses, interpretations, or conclusions.

\section{AUTHOR CONTRIBUTIONS}

The following authors participated in study design (JL, RG, $\mathrm{CL}$ ), data processing and analysis (EPW, KR, MF, RH, JL), and manuscript writing (JL, CL). All authors reviewed, commented, and approved the manuscript.

\section{REFERENCES}

Andrews-Hanna JR, Reidler JS, Sepulcre J, Poulin R, Buckner RL (2010). Functional-anatomic fractionation of the brain's default network. Neuron 65: 550-562.

Arnsten AF, Jin LE (2014). Molecular influences on working memory circuits in dorsolateral prefrontal cortex. Prog $\mathrm{Mol} \mathrm{Biol}$ Transl Sci 122: 211-231.

Ashare RL, Falcone M, Lerman C (2014). Cognitive function during nicotine withdrawal: implications for nicotine dependence treatment. Neuropharmacology 76(Pt B): 581-591.

Ashare RL, Wileyto EP, Perkins KA, Schnoll RA (2013a). The first 7 days of a quit attempt predicts relapse: validation of a measure for screening medications for nicotine dependence. J Addict Med 7: 249-254.

Ashare RL, Wileyto EP, Ruparel K, Goelz PM, Hopson RD, Valdez JN et al (2013b). Effects of tolcapone on working memory and brain activity in abstinent smokers: a proof-of-concept study. Drug Alcohol Depend 133: 852-856.

Austin PC, Tu JV (2004a). Automated variable selection methods for logistic regression produced unstable models for predicting acute myocardial infarction mortality. J Clin Epidemiol 57: $1138-1146$.

Austin PC, Tu JV (2004b). Bootstrap methods for developing predictive models. Am Stat 58: 131-137.

Baddeley A (2003). Working memory: looking back and looking forward. Nat Rev Neurosci 4: 829-839.

Beaver JD, Long CJ, Cole DM, Durcan MJ, Bannon LC, Mishra RG et al (2011). The effects of nicotine replacement on cognitive brain activity during smoking withdrawal studied with simultaneous fMRI/EEG. Neuropsychopharmacology 36: 1792-1800.
Botvinick MM, Braver TS, Barch DM, Carter CS, Cohen JD (2001). Conflict monitoring and cognitive control. Psychol Rev 108: 624-652.

Bough KJ, Lerman C, Rose JE, McClernon FJ, Kenny PJ, Tyndale RF et al (2013). Biomarkers for smoking cessation. Clin Pharmacol Therap 93: 526-538.

Bradley A (1997). The use of the area under the ROC curve in the evaluation of machine learning algorithms. Pattern Recognit 30: 1145-1159.

Brown R, Burgess E, Sales S, Whiteley J (1998). Reliability and validity of a smoking timeline follow-back interview. Addict Behav 12: 101-112.

Brozoski TJ, Brown RM, Rosvold HE, Goldman PS (1979). Cognitive deficit caused by regional depletion of dopamine in prefrontal cortex of rhesus monkey. Science 205: 929-932.

Cox LS, Tiffany ST, Christen AG (2001). Evaluation of the brief questionnaire of smoking urges (QSU-brief) in laboratory and clinical settings. Nicotine Tob Res 3: 7-16.

Croxson PL, Kyriazis DA, Baxter MG (2011). Cholinergic modulation of a specific memory function of prefrontal cortex. Nat Neurosci 14: 1510-1512.

Dresler M, Sandberg A, Ohla K, Bublitz C, Trenado C, MroczkoWasowicz A et al (2013). Non-pharmacological cognitive enhancement. Neuropharmacology 64: 529-543.

Falcone M, Wileyto EP, Ruparel K, Gerraty RT, LaPrate L, Detre JA et al (2014). Age-related differences in working memory deficits during nicotine withdrawal. Addict Biol 19: 907-917.

Fassbender C, Murphy K, Foxe JJ, Wylie GR, Javitt DC, Robertson IH et al (2004). A topography of executive functions and their interactions revealed by functional magnetic resonance imaging. Brain Res Cogn Brain Res 20: 132-143.

Fawcett T (2003). ROC Graphs: Notes and practical considerations for researchers. Technical Report, HPL-2003-4, HP Laboratories.

Filevich E, Kuhn S, Haggard P (2012). Intentional inhibition in human action: the power of 'no'. Neurosci Biobehav Rev 36: $1107-1118$

Fox MD, Snyder AZ, Vincent JL, Corbetta M, Van Essen DC, Raichle ME (2005). The human brain is intrinsically organized into dynamic, anticorrelated functional networks. PNAS 102: 9673-9678.

Gonzales MM, Tarumi T, Miles SC, Tanaka H, Shah F, Haley AP (2010). Insulin sensitivity as a mediator of the relationship between BMI and working memory-related brain activation. Obesity 18: 2131-2137.

Gourlay SG, Forbes A, Marriner T, Pethica D, McNeil JJ (1994). Prospective study of factors predicting outcome of transdermal nicotine treatment in smoking cessation. BMJ 309: 842-846.

Gusnard DA, Akbudak E, Shulman GL, Raichle ME (2001). Medial prefrontal cortex and self-referential mental activity: relation to a default mode of brain function. PNAS 98: 4259-4264.

Hare TA, Camerer CF, Rangel A (2009). Self-control in decisionmaking involves modulation of the vmPFC valuation system. Science 324: 646-648.

Harrell FE Jr., Lee KL, Mark DB (1996). Multivariable prognostic models: issues in developing models, evaluating assumptions and adequacy, and measuring and reducing errors. Stat Med 15: 361-387.

Heatherton TF, Kozlowski LT, Frecker RC, Fagerstrom KO (1991). The Fagerstrom Test for Nicotine Dependence: a revision of the Fagerstrom Tolerance Questionnaire. Br J Addict 86: 1119-1127.

Hege MA, Stingl KT, Ketterer C, Haring HU, Heni M, Fritsche A et al (2013). Working memory-related brain activity is associated with outcome of lifestyle intervention. Obesity 21: 2488-2494.

Hosmer D, Lemeshow S (2000). Applied Logistic Regression. 2nd edn, John Wiley \& Sons: New York, NY, USA.

Hughes JR, Hatsukami D (1986). Signs and symptoms of tobacco withdrawal. Arch Gen Psychiatry 43: 289-294. 
Hughes JR, Keely J, Naud S (2004). Shape of the relapse curve and long-term abstinence among untreated smokers. Addiction 99: 29-38.

Hymowitz N, Cummings KM, Hyland A, Lynn WR, Pechacek TF, Hartwell TD (1997). Predictors of smoking cessation in a cohort of adult smokers followed for five years. Tob Control 6(Suppl 2): S57-S62.

Jacobsen LK, Mencl WE, Constable RT, Westerveld M, Pugh KR (2007). Impact of smoking abstinence on working memory neurocircuitry in adolescent daily tobacco smokers. Psychopharmacology 193: 557-566.

Japuntich SJ, Leventhal AM, Piper ME, Bolt DM, Roberts LJ, Fiore MC et al (2011). Smoker characteristics and smokingcessation milestones. Am J Prev Med 40: 286-294.

Jenkinson M, Bannister P, Brady M, Smith S (2002). Improved optimization for the robust and accurate linear registration and motion correction of brain images. Neurolmage 17: 825-841.

Jenkinson M, Smith S (2001). A global optimisation method for robust affine registration of brain images. Med Image Anal 5: 143-156.

Kollins SH, English JS, Roley ME, O'Brien B, Blair J, Lane SD et al (2013). Effects of smoking abstinence on smoking-reinforced responding, withdrawal, and cognition in adults with and without attention deficit hyperactivity disorder. Psychopharmacology 227: 19-30.

Kouneiher F, Charron S, Koechlin E (2009). Motivation and cognitive control in the human prefrontal cortex. Nat Neurosci 12: $939-945$.

Krain AL, Wilson AM, Arbuckle R, Castellanos FX, Milham MP (2006). Distinct neural mechanisms of risk and ambiguity: a meta-analysis of decision-making. NeuroImage 32: 477-484.

Lerman C, Gu H, Loughead J, Ruparel K, Yang Y, Stein EA (2014). Large-scale brain network coupling predicts acute nicotine abstinence effects on craving and cognitive function. JAMA Psychiatry 71: 523-530.

Lerman C, Kaufmann V, Rukstalis M, Patterson F, Perkins K, Audrain-McGovern $\mathrm{J}$ et al (2004). Individualizing nicotine replacement therapy for the treatment of tobacco dependence: a randomized trial. Ann Intern Med 140: 426-433.

Loughead J, Ray R, Wileyto EP, Ruparel K, Sanborn P, Siegel S et al (2010). Effects of the alpha4beta2 partial agonist varenicline on brain activity and working memory in abstinent smokers. Biol Psychiatry 67: 715-721.

Loughead J, Wileyto EP, Valdez JN, Sanborn P, Tang K, Strasser AA et al (2009). Effect of abstinence challenge on brain function and cognition in smokers differs by COMT genotype. Mol Psychiatry 14: 820-826.

MacDonald AW 3rd, Cohen JD, Stenger VA, Carter CS (2000). Dissociating the role of the dorsolateral prefrontal and anterior cingulate cortex in cognitive control. Science 288: 1835-1838.

Mendrek A, Monterosso J, Simon SL, Jarvik M, Brody A, Olmstead $\mathrm{R}$ et al (2006). Working memory in cigarette smokers: comparison to non-smokers and effects of abstinence. Addict Behav 31: 833-844.

Myers CS, Taylor RC, Moolchan ET, Heishman SJ (2008). Doserelated enhancement of mood and cognition in smokers administered nicotine nasal spray. Neuropsychopharmacology 33: $588-598$.

Nestor L, McCabe E, Jones J, Clancy L, Garavan H (2011). Differences in "bottom-up" and "top-down" neural activity in current and former cigarette smokers: Evidence for neural substrates which may promote nicotine abstinence through increased cognitive control. NeuroImage 56: 2258-2275.

Niendam TA, Laird AR, Ray KL, Dean YM, Glahn DC, Carter CS (2012). Meta-analytic evidence for a superordinate cognitive control network subserving diverse executive functions. Cogn Affect Behav Neurosci 12: 241-268.
Nocente R, Vitali M, Balducci G, Enea D, Kranzler HR, Ceccanti M (2013). Varenicline and neuronal nicotinic acetylcholine receptors: a new approach to the treatment of co-occurring alcohol and nicotine addiction? Am J Addict 22: 453-459.

Owen AM, McMillan KM, Laird AR, Bullmore E (2005). N-back working memory paradigm: a meta-analysis of normative functional neuroimaging studies. Hum Brain Mapp 25: 46-59.

Patterson F, Jepson C, Loughead J, Perkins K, Strasser AA, Siegel S et al (2010). Working memory deficits predict short-term smoking resumption following brief abstinence. Drug Alcohol Depend 106: 61-64.

Perkins KA, Karelitz JL, Jao NC, Gur RC, Lerman C (2013). Effects of bupropion on cognitive performance during initial tobacco abstinence. Drug Alcohol Depend 133: 283-286.

Piasecki TM (2006). Relapse to smoking. Clin Psychol Rev 26: 196-215.

Ragland JD, Turetsky BI, Gur RC, Gunning-Dixon F, Turner T, Schroeder L et al (2002). Working memory for complex figures: an fMRI comparison of letter and fractal n-back tasks. Neuropsychology 16: 370-379.

Raichle ME, MacLeod AM, Snyder AZ, Powers WJ, Gusnard DA, Shulman GL (2001). A default mode of brain function. PNAS 98: 676-682.

Satterthwaite TD, Wolf DH, Erus G, Ruparel K, Elliott MA, Gennatas ED et al (2013). Functional maturation of the executive system during adolescence. J Neurosci 33: 16249-16261.

Segrave RA, Arnold S, Hoy K, Fitzgerald PB (2014). Concurrent cognitive control training augments the antidepressant efficacy of tDCS: a pilot study. Brain Stim 7: 325-331.

Sheehan DV, Lecrubier Y, Sheehan KH, Amorim P, Janavs J, Weiller E et al (1998). The Mini-International Neuropsychiatric Interview (M.I.N.I.): the development and validation of a structured diagnostic psychiatric interview for DSM-IV and ICD-10. J Clin Psychiaty 59(Suppl 20): 22-33; quiz 34-57.

Sheffer CE, Mennemeier M, Landes RD, Bickel WK, Brackman S, Dornhoffer J et al (2013). Neuromodulation of delay discounting, the reflection effect, and cigarette consumption. J Subst Abuse Treat 45: 206-214.

Smith SM (2002). Fast robust automated brain extraction. Hum Brain Mapp 17: 143-155.

Smith SM, Fox PT, Miller KL, Glahn DC, Fox PM, Mackay CE et al (2009). Correspondence of the brain's functional architecture during activation and rest. PNAS 106: 13040-13045.

Snowball A, Tachtsidis I, Popescu T, Thompson J, Delazer M, Zamarian L et al (2013). Long-term enhancement of brain function and cognition using cognitive training and brain stimulation. Current Biol 23: 987-992.

Steyerberg EW (2008). Clinical Prediction Models: A Practical Approach to Development, Validation, and Updating. Springer: New York, NY, USA.

Sutherland MT, Carroll AJ, Salmeron BJ, Ross TJ, Hong LE, Stein EA (2013). Down-regulation of amygdala and insula functional circuits by varenicline and nicotine in abstinent cigarette smokers. Biol Psychiatry 74: 538-546.

Sutherland MT, McHugh MJ, Pariyadath V, Stein EA (2012). Resting state functional connectivity in addiction: lessons learned and a road ahead. NeuroImage 62: 2281-2295.

Sweitzer MM, Denlinger RL, Donny EC (2013). Dependence and withdrawal-induced craving predict abstinence in an incentivebased model of smoking relapse. Nicotine Tob Res 15: 36-43.

Talairach J, Tournoux P (1988). Co-planar stereotaxic atlas of the human brain. Thieme, New York.

UDHHS (2006). Clearing the Air. U.S. Department of Health and Human Services, National Institutes of Health, National Cancer Institute. NIH Publication No. 08-1647.

Watson D, Clark LA, Tellegen A (1988). Development and validation of brief measures of positive and negative affect: the PANAS scales. J Personal Soc Psychol 54: 1063-1070. 
Whelan R, Garavan H (2014). When optimism hurts: inflated predictions in psychiatric neuroimaging. Biol Psychiatry 75: 746-748.

Wing VC, Barr MS, Wass CE, Lipsman N, Lozano AM, Daskalakis ZJ et al (2013). Brain stimulation methods to treat tobacco addiction. Brain Stim 6: 221-230.
Zachary RA (1986). Shipley Institute of Living Scale: Revised Manual. Western Psychological Services: Los Angeles, CA, USA. Zhang G, Yao L, Zhang H, Long Z, Zhao X (2013). Improved working memory performance through self-regulation of dorsal lateral prefrontal cortex activation using real-time fMRI. PloS One 8: e73735. 